J. Korean Math. Soc. 51 (2014), No. 4, pp. 791-815

http://dx.doi.org/10.4134/JKMS.2014.51.4.791

\title{
INEQUALITIES FOR THE RIEMANN-STIELTJES INTEGRAL OF PRODUCT INTEGRATORS WITH APPLICATIONS
}

\author{
Silvestru Sever Dragomir
}

Abstract. We show amongst other that if $f, g:[a, b] \rightarrow \mathbb{C}$ are two functions of bounded variation and such that the Riemann-Stieltjes integral $\int_{a}^{b} f(t) d g(t)$ exists, then for any continuous functions $h:[a, b] \rightarrow \mathbb{C}$, the Riemann-Stieltjes integral $\int_{a}^{b} h(t) d(f(t) g(t))$ exists and

$$
\int_{a}^{b} h(t) d(f(t) g(t))=\int_{a}^{b} h(t) f(t) d(g(t))+\int_{a}^{b} h(t) g(t) d(f(t)) .
$$

Using this identity we then provide sharp upper bounds for the quantity

$$
\left|\int_{a}^{b} h(t) d(f(t) g(t))\right|
$$

and apply them for trapezoid and Ostrowski type inequalities. Some applications for continuous functions of selfadjoint operators on complex Hilbert spaces are given as well.

\section{Introduction}

One of the most important properties of the Riemann-Stieltjes integral

$$
\int_{a}^{b} f(t) d g(t)
$$

is the fact that this integral exists if one of the function is of bounded variation while the other is continuous. The following sharp inequality holds

$$
\left|\int_{a}^{b} f(t) d g(t)\right| \leq \max _{t \in[a, b]}|f(t)| \bigvee_{a}^{b}(g),
$$

provided that $f:[a, b] \rightarrow \mathbb{C}$ is continuous on $[a, b]$ and $g:[a, b] \rightarrow \mathbb{C}$ is of bounded variation on this interval. Here $\bigvee^{b}(g)$ denotes the total variation of $g$ on $[a, b]$.

Received November 20, 2013; Revised March 5, 2014.

2010 Mathematics Subject Classification. 26D15, $47 \mathrm{~A} 63$.

Key words and phrases. Riemann-Stieltjes integral, functions of bounded variation, Trapezoid and midpoint inequalities, selfadjoint operators, functions of selfadjoint operators. 
When $g$ is Lipschitzian with the constant $L>0$, i.e.,

$$
|g(t)-g(s)| \leq L|t-s|
$$

for any $t, s \in[a, b]$, then we have

$$
\left|\int_{a}^{b} f(t) d g(t)\right| \leq L \int_{a}^{b}|f(t)| d t
$$

for any Riemann integrable function $f:[a, b] \rightarrow \mathbb{C}$.

Moreover, if the integrator $g$ is monotonic nondecreasing on the interval $[a, b]$ and $f:[a, b] \rightarrow \mathbb{C}$ is continuous, then we have the modulus inequality

$$
\left|\int_{a}^{b} f(t) d g(t)\right| \leq \int_{a}^{b}|f(t)| d g(t) .
$$

The above inequalities have been used by many authors to derive various integral inequalities. We provide here some simple examples.

The following generalized trapezoidal inequality for the function of bounded variation $f:[a, b] \rightarrow \mathbb{C}$ was obtained in 1999 by the author [21, Proposition 1]

$$
\begin{aligned}
& \left|\int_{a}^{b} f(t) d t-(x-a) f(a)-(b-x) f(b)\right| \\
\leq & {\left[\frac{1}{2}(b-a)+\left|x-\frac{a+b}{2}\right|\right] \bigvee_{a}^{b}(f), }
\end{aligned}
$$

where $x \in[a, b]$. The constant $\frac{1}{2}$ cannot be replaced by a smaller quantity. See also [19] for a different proof and other details.

The best inequality one can derive from (1.4) is the trapezoid inequality

$$
\left|\int_{a}^{b} f(t) d t-\frac{f(a)+f(b)}{2}(b-a)\right| \leq \frac{1}{2}(b-a) \bigvee_{a}^{b}(f) .
$$

Here the constant $\frac{1}{2}$ is also best possible.

For related results, see [11]-[15], [17]-[20], [24]-[25], [29]-[32], [35], [41], [34], [43]-[45] and [53]-[55].

In order to extend the classical Ostrowski's inequality for differentiable functions with bounded derivatives to the larger class of functions of bounded variation, the author obtained in 1999 (see [21] or the RGMIA preprint version of [23]) the following result

$$
\left|\int_{a}^{b} f(t) d t-f(x)(b-a)\right| \leq\left[\frac{1}{2}(b-a)+\left|x-\frac{a+b}{2}\right|\right] \bigvee_{a}^{b}(f)
$$

for any $x \in[a, b]$ and $f:[a, b] \rightarrow \mathbb{C}$ a function of bounded variation on $[a, b]$. Here $\bigvee_{a}^{b}(f)$ denotes the total variation of $f$ on $[a, b]$ and the constant $\frac{1}{2}$ is best 
possible in (1.6). The best inequality one can obtain from (1.6) is the midpoint inequality, namely

$$
\left|\int_{a}^{b} f(t) d t-f\left(\frac{a+b}{2}\right)(b-a)\right| \leq \frac{1}{2}(b-a) \bigvee_{a}^{b}(f)
$$

for which the constant $\frac{1}{2}$ is also sharp.

For related results, see [1]-[11], [16]-[17], [21], [23], [25]-[27], [31], [36]-[38], [42], [46]-[52] and [56]-[59].

Motivated by the above results, we establish in this paper bounds for the quantity

$$
\left|\int_{a}^{b} h(t) d(f(t) g(t))\right|
$$

in the case when the integrand $h$ is continuous while the functions $f$ and $g$ are of bounded variation. Applications for the trapezoidal and midpoint inequalities are given. Some applications for continuous functions of selfadjoint operators on complex Hilbert spaces are provided as well.

\section{The main results}

The following identity is of interest in itself:

Lemma 1. Let $f, g:[a, b] \rightarrow \mathbb{C}$ be two functions of bounded variation and such that the Riemann-Stieltjes integral $\int_{a}^{b} f(t) d g(t)$ exists. If $h:[a, b] \rightarrow \mathbb{C}$ is continuous, then the Riemann-Stieltjes integral $\int_{a}^{b} h(t) d(f(t) g(t))$ exists and

$$
\int_{a}^{b} h(t) d(f(t) g(t))=\int_{a}^{b} h(t) f(t) d(g(t))+\int_{a}^{b} h(t) g(t) d(f(t)) .
$$

Proof. Since $f, g:[a, b] \rightarrow \mathbb{C}$ are of bounded variation, then $f g$ is of bounded variation and since $h:[a, b] \rightarrow \mathbb{C}$ is continuous, it follows that the RiemannStieltjes integral $\int_{a}^{b} h(t) d(f(t) g(t))$ exists.

Observe that, since the integral $\int_{a}^{b} f(t) d g(t)$ exists, then for any $s \in[a, b]$ the integral $\ell(s):=\int_{a}^{s} f(t) d g(t)$ exists and the function $\ell$ is of bounded variation on $[a, b]$.

Indeed, let

$$
a=s_{0}<s_{1}<\cdots<s_{n-1}<s_{n}=b
$$

a division of the interval $[a, b]$. Then we have

$$
\begin{aligned}
\sum_{i=0}^{n-1}\left|\ell\left(s_{i+1}\right)-\ell\left(s_{i}\right)\right| & =\sum_{i=0}^{n-1}\left|\int_{a}^{s_{i+1}} f(t) d g(t)-\int_{a}^{s_{i}} f(t) d g(t)\right| \\
& =\sum_{i=0}^{n-1}\left|\int_{s_{i}}^{s_{i+1}} f(t) d g(t)\right| \leq \sum_{i=0}^{n-1}\left(\sup _{t \in\left[s_{i}, s_{i+1}\right]}|f(t)| \bigvee_{s_{i}}^{s_{i+1}}(g)\right)
\end{aligned}
$$




$$
\leq \sup _{t \in[a, b]}|f(t)| \sum_{i=0}^{n-1}\left(\bigvee_{s_{i}}^{s_{i+1}}(g)\right)=\sup _{t \in[a, b]}|f(t)| \bigvee_{a}^{b}(g)<\infty
$$

which shows that $\ell$ is of bounded variation on $[a, b]$ and

$$
\bigvee_{a}^{b}(\ell) \leq\|f\|_{\infty} \bigvee_{a}^{b}(g)
$$

where $\|f\|_{\infty}:=\sup _{t \in[a, b]}|f(t)|$.

Now, by the integration by parts theorem, since $\int_{a}^{s} f(t) d g(t)$ exists for any $s \in[a, b]$, then $\int_{a}^{s} g(t) d f(t)$ also exists and we have the equality

$$
f(s) g(s)=f(a) g(a)+\int_{a}^{s} f(t) d g(t)+\int_{a}^{s} g(t) d f(t)
$$

for any $s \in[a, b]$.

Since the functions $\int_{a}^{\cdot} f(t) d g(t)$ and $\int_{a}^{\cdot} g(t) d f(t)$ are of bounded variation, then the Riemann-Stieltjes integrals

$$
\int_{a}^{b} h(s) d\left(\int_{a}^{s} f(t) d g(t)\right) \text { and } \int_{a}^{b} h(s) d\left(\int_{a}^{s} g(t) d f(t)\right)
$$

exist and

$$
\int_{a}^{b} h(s) d\left(\int_{a}^{s} f(t) d g(t)\right)=\int_{a}^{b} h(s) f(s) d g(s)
$$

and

$$
\int_{a}^{b} h(s) d\left(\int_{a}^{s} g(t) d f(t)\right)=\int_{a}^{b} h(s) g(s) d f(s) .
$$

Now, on utilizing (2.2), (2.3) and (2.4) we have

$$
\begin{aligned}
\int_{a}^{b} h(s) d(f(s) g(s))= & \int_{a}^{b} h(s) d(f(a) g(a)) \\
& +\int_{a}^{b} h(s) d\left(\int_{a}^{s} f(t) d g(t)\right) \\
& +\int_{a}^{b} h(s)\left(\int_{a}^{s} g(t) d f(t)\right) \\
= & \int_{a}^{b} h(s) f(s) d g(s)+\int_{a}^{b} h(s) g(s) d f(s)
\end{aligned}
$$

and the equality (2.1) is proved.

Remark 1 . The dual case also holds, namely, when the functions $f, g:[a, b] \rightarrow \mathbb{C}$ are continuous and such that the Riemann-Stieltjes integral $\int_{a}^{b} f(t) d g(t)$ exists, then for any function $h:[a, b] \rightarrow \mathbb{C}$ of bounded variation the Riemann-Stieltjes integral $\int_{a}^{b} h(t) d(f(t) g(t))$ exists and the equality (2.1) is satisfied. 
Corollary 1. Let $f:[a, b] \rightarrow \mathbb{C}$ be a functions of bounded variation and such that the Riemann-Stieltjes integral $\int_{a}^{b} f(t) d f(t)$ exists. If $h:[a, b] \rightarrow \mathbb{C}$ is continuous, then the Riemann-Stieltjes integral $\int_{a}^{b} h(t) d\left(f^{2}(t)\right)$ exists and

$$
\int_{a}^{b} h(t) d\left(f^{2}(t)\right)=2 \int_{a}^{b} h(t) f(t) d(f(t)) .
$$

If $\int_{a}^{b} f(t) d \overline{f(t)}$ exists, then for any continuous function $h:[a, b] \rightarrow \mathbb{C}$, the Riemann-Stieltjes integral $\int_{a}^{b} h(t) d\left(|f(t)|^{2}\right)$ exists and

$$
\int_{a}^{b} h(t) d\left(|f(t)|^{2}\right)=\int_{a}^{b} h(t) f(t) d(\overline{f(t)})+\int_{a}^{b} h(t) \overline{f(t)} d(f(t)) .
$$

In particular, if $h:[a, b] \rightarrow \mathbb{R}$, then

$$
\int_{a}^{b} h(t) d\left(|f(t)|^{2}\right)=2 \operatorname{Re}\left(\int_{a}^{b} h(t) \overline{f(t)} d(f(t))\right) .
$$

The first bound for the Riemann-Stieltjes integral of product integrators is as follows:

Theorem 1. Let $f, g:[a, b] \rightarrow \mathbb{C}$ be two functions of bounded variation and such that the Riemann-Stieltjes integral $\int_{a}^{b} f(t) d g(t)$ exists. If $h:[a, b] \rightarrow \mathbb{C}$ is continuous, then

$$
\begin{aligned}
\left|\int_{a}^{b} h(t) d(f(t) g(t))\right| & \leq\|h f\|_{\infty} \bigvee_{a}^{b}(g)+\|h g\|_{\infty} \bigvee_{a}^{b}(f) \\
& \leq\|h\|_{\infty}\left[\|f\|_{\infty} \bigvee_{a}^{b}(g)+\|g\|_{\infty} \bigvee_{a}^{b}(f)\right]
\end{aligned}
$$

Both inequalities in (2.8) are sharp.

Proof. We know that if $p:[a, b] \rightarrow \mathbb{C}$ is bounded, $v:[a, b] \rightarrow \mathbb{C}$ is of bounded variation and the Riemann-Stieltjes integral $\int_{a}^{b} p(s) d v(s)$ exists, then we have the inequality

$$
\left|\int_{a}^{b} p(s) d v(s)\right| \leq\|p\|_{\infty} \bigvee_{a}^{b}(v),
$$

where $\|p\|_{\infty}=\sup _{t \in[a, b]}|p(t)|<\infty$.

Taking the modulus in (2.1) and using the property (2.9) we have

$$
\begin{aligned}
\left|\int_{a}^{b} h(t) d(f(t) g(t))\right| & \leq\left|\int_{a}^{b} h(t) f(t) d(g(t))\right|+\left|\int_{a}^{b} h(t) g(t) d(f(t))\right| \\
& \leq\|h f\|_{\infty} \bigvee_{a}^{b}(g)+\|h g\|_{\infty} \bigvee_{a}^{b}(f)
\end{aligned}
$$




$$
\begin{aligned}
& \leq\|h\|_{\infty}\|f\|_{\infty} \bigvee_{a}^{b}(g)+\|h\|_{\infty}\|g\|_{\infty} \bigvee_{a}^{b}(f) \\
& =\|h\|_{\infty}\left[\|f\|_{\infty} \bigvee_{a}^{b}(g)+\|g\|_{\infty} \bigvee_{a}^{b}(f)\right]
\end{aligned}
$$

and the inequality $(2.8)$ is proved.

Now, to prove the sharpness of the inequalities (2.8) we consider the functions $f, g:[a, b] \rightarrow \mathbb{R}$ given by

$$
f(t):=\left\{\begin{array}{ll}
0 & \text { if } \quad t=a \\
1 & \text { if } \quad t \in(a, b],
\end{array} \quad g(t):=\left\{\begin{array}{lll}
1 & \text { if } \quad t \in[a, b) \\
0 & \text { if } \quad t=b .
\end{array}\right.\right.
$$

The functions $f$ and $g$ are of bounded variation, $\bigvee_{a}^{b}(f)=\bigvee_{a}^{b}(g)=1$ and $\|f\|_{\infty}=$ $\|g\|_{\infty}=1$. We have

$$
f(t) g(t):= \begin{cases}0 & \text { if } t=a \\ 1 & \text { if } t \in(a, b) \\ 0 & \text { if } t=b .\end{cases}
$$

The function $f g$ is of bounded variation and for a continuous function $h$ : $[a, b] \rightarrow \mathbb{C}$ the Riemann-Stieltjes integral $\int_{a}^{b} h(t) d(f(t) g(t))$ exists and integrating by parts we have

$$
\begin{aligned}
& \int_{a}^{b} h(t) d(f(t) g(t)) \\
= & f(b) g(b) h(b)-f(a) g(a) h(a)-\int_{a}^{b} f(t) g(t) d(h(t)) \\
= & -\int_{a}^{b} f(t) g(t) d(h(t)) .
\end{aligned}
$$

Consider the following sequence of divisions and intermediate points

$$
a=x_{0}^{(n)}<\xi_{0}^{(n)}<x_{1}^{(n)}<\cdots<x_{n-1}^{(n)}<\xi_{n-1}^{(n)}<x_{n}^{(n)}=b
$$

such that the norm of the division $\Delta_{n}:=\max _{i \in\{0, \ldots, n-1\}}\left(x_{i+1}^{(n)}-x_{i}^{(n)}\right) \rightarrow 0$ as $n \rightarrow \infty$. By the definition of the Riemann-Stieltjes integral we have

$$
\begin{aligned}
\int_{a}^{b} f(t) g(t) d(h(t)) & =\lim _{n \rightarrow \infty} \sum_{i=0}^{n-1} f\left(\xi_{i}^{(n)}\right) g\left(\xi_{i}^{(n)}\right)\left(h\left(x_{i+1}^{(n)}\right)-h\left(x_{i}^{(n)}\right)\right) \\
& =\lim _{n \rightarrow \infty} \sum_{i=0}^{n-1}\left(h\left(x_{i+1}^{(n)}\right)-h\left(x_{i}^{(n)}\right)\right)=h(b)-h(a),
\end{aligned}
$$

and then, by (2.10) we have

$$
\int_{a}^{b} h(t) d(f(t) g(t))=-h(b)+h(a) .
$$


We also have

$$
h(t) f(t):=\left\{\begin{array}{cl}
0 & \text { if } t=a \\
h(t) & \text { if } t \in(a, b],
\end{array} \quad h(t) g(t):=\left\{\begin{array}{cl}
h(t) & \text { if } t \in[a, b) \\
0 & \text { if } t=b,
\end{array}\right.\right.
$$

which implies that

$$
\|h f\|_{\infty}=\|h g\|_{\infty}=\|h\|_{\infty} .
$$

Therefore the inequality $(2.8)$ reduces to

$$
|h(b)-h(a)| \leq 2\|h\|_{\infty} \leq 2\|h\|_{\infty} .
$$

We observe that, this inequality is sharp since for continuous functions $h$ : $[a, b] \rightarrow \mathbb{R}$ for which

$$
0<h(b)=-h(a)=\sup _{t \in[a, b]} h(t),
$$

we get equality in (2.11).

For instance, if we take

$$
h(t)=t-\frac{a+b}{2}, t \in[a, b],
$$

then

$$
|h(b)-h(a)|=b-a,\|h\|_{\infty}=\frac{b-a}{2}
$$

and the equality in (2.11) is realized.

Remark 2. We observe that if one of the functions $f$ or $g$ is constant, then (2.8) reduces to $(1.1)$

We say that the function $f:[a, b] \rightarrow \mathbb{C}$ is Lipschitzian with the constant $L>0$ if

$$
|f(t)-f(s)| \leq L|t-s|
$$

for any $t, s \in[a, b]$.

Theorem 2. Assume that the function $f:[a, b] \rightarrow \mathbb{C}$ is Lipschitzian with the constant $L>0, g:[a, b] \rightarrow \mathbb{C}$ is Lipschitzian with the constant $K>0$ and $h:[a, b] \rightarrow \mathbb{C}$ a continuous function on $[a, b]$. Then we have the inequality

$$
\begin{aligned}
\left|\int_{a}^{b} h(t) d(f(t) g(t))\right| & \leq K \int_{a}^{b}|h(t) f(t)| d t+L \int_{a}^{b}|h(t) g(t)| d t \\
& \leq \max \{K, L\} \int_{a}^{b}|h(t)|(|f(t)|+|g(t)|) d t
\end{aligned}
$$

The inequalities in (2.12) are sharp. 
Proof. It is known that, if $p:[a, b] \rightarrow \mathbb{C}$ is continuous and $v:[a, b] \rightarrow \mathbb{C}$ is Lipschitzian with the constant $L>0$, then the Riemann-Stieltjes integral $\int_{a}^{b} p(s) d v(s)$ exists and we have the inequality

$$
\left|\int_{a}^{b} p(s) d v(s)\right| \leq L \int_{a}^{b}|p(s)| d s .
$$

Taking the modulus in (2.1) and using the property (2.9) we have

$$
\begin{aligned}
\left|\int_{a}^{b} h(t) d(f(t) g(t))\right| & \leq\left|\int_{a}^{b} h(t) f(t) d(g(t))\right|+\left|\int_{a}^{b} h(t) g(t) d(f(t))\right| \\
& \leq K \int_{a}^{b}|h(t) f(t)| d t+L \int_{a}^{b}|h(t) g(t)| d t \\
& \leq \max \{K, L\} \int_{a}^{b}|h(t)|(|f(t)|+|g(t)|) d t
\end{aligned}
$$

and the inequality (2.12) is proved.

Consider now the functions $f, g:[a, b] \rightarrow \mathbb{R}, f(t)=g(t)=\left|t-\frac{a+b}{2}\right|$. We observe that $f$ and $g$ are Lipschitzian with the constant $L=1$.

Indeed, for any $t, s \in[a, b]$ we have

$$
\begin{aligned}
|f(t)-f(s)| & =|| t-\frac{a+b}{2}|-| s-\frac{a+b}{2}|| \\
& \leq|t-s|,
\end{aligned}
$$

which shows that the function $f$ is Lipschitzian with the constant $L=1$.

Now

$$
\begin{aligned}
\left|\int_{a}^{b} h(t) d(f(t) g(t))\right| & =\int_{a}^{b} h(t) d\left(\left(t-\frac{a+b}{2}\right)^{2}\right) \\
& =2\left|\int_{a}^{b} h(t)\left(t-\frac{a+b}{2}\right) d t\right|
\end{aligned}
$$

and

$$
K \int_{a}^{b}|h(t) f(t)| d t+L \int_{a}^{b}|h(t) g(t)| d t=2 \int_{a}^{b}|h(t)|\left|t-\frac{a+b}{2}\right| d t
$$

and the first inequality in (2.12) becomes

$$
\left|\int_{a}^{b} h(t)\left(t-\frac{a+b}{2}\right) d t\right| \leq \int_{a}^{b}|h(t)|\left|t-\frac{a+b}{2}\right| d t .
$$

We observe that the equality case holds if we take $h:[a, b] \rightarrow \mathbb{R}, h(t)=$ $t-\frac{a+b}{2}$. 
Remark 3. We remark that in the dual case, namely when the functions $f, g$ : $[a, b] \rightarrow \mathbb{C}$ are continuous and $h:[a, b] \rightarrow \mathbb{C}$ is Lipschitzian with a constant $M>0$, then the Riemann-Stieltjes integral $\int_{a}^{b} h(t) d(f(t) g(t))$ exists and integrating by parts we have

$\int_{a}^{b} h(t) d(f(t) g(t))=f(b) g(b) h(b)-f(a) g(a) h(a)-\int_{a}^{b} f(t) g(t) d(h(t))$ or, equivalently

$$
\begin{aligned}
& f(b) g(b) h(b)-f(a) g(a) h(a)-\int_{a}^{b} h(t) d(f(t) g(t)) \\
= & \int_{a}^{b} f(t) g(t) d(h(t)) .
\end{aligned}
$$

Taking the modulus in (2.14) and using the property (2.13) we get the inequality $\left|f(b) g(b) h(b)-f(a) g(a) h(a)-\int_{a}^{b} h(t) d(f(t) g(t))\right| \leq M \sup _{t \in[a, b]}|f(t) g(t)|$.

Theorem 3. Assume that $f, g:[a, b] \rightarrow \mathbb{R}$ are monotonic nondecreasing on $[a, b]$ and such that the Riemann-Stieltjes integral $\int_{a}^{b} f(t) d g(t)$ exists, and $h$ : $[a, b] \rightarrow \mathbb{C}$ is continuous on $[a, b]$. Then we have the inequality

$$
\left|\int_{a}^{b} h(t) d(f(t) g(t))\right| \leq \int_{a}^{b}|f(t) h(t)| d g(t)+\int_{a}^{b}|g(t) h(t)| d f(t) .
$$

The inequality (2.15) is sharp.

Proof. It is well known that if $p:[a, b] \rightarrow \mathbb{C}$ is continuous and $v:[a, b] \rightarrow \mathbb{R}$ is monotonic nondecreasing, then the Riemann-Stieltjes integral $\int_{a}^{b} p(t) d v(t)$ exists and

$$
\left|\int_{a}^{b} p(t) d v(t)\right| \leq \int_{a}^{b}|p(t)| d v(t) .
$$

Taking the modulus in (2.1) we have

$$
\begin{aligned}
\left|\int_{a}^{b} h(t) d(f(t) g(t))\right| & \leq\left|\int_{a}^{b} f(t) h(t) d g(t)\right|+\left|\int_{a}^{b} g(t) h(t) d f(t)\right| \\
& \leq \int_{a}^{b}|f(t) h(t)| d g(t)+\int_{a}^{b}|g(t) h(t)| d f(t) .
\end{aligned}
$$

Consider the functions $f, g:[a, b] \rightarrow \mathbb{R}$ defined by

$$
f(t):=\left\{\begin{array}{ll}
0 & \text { if } t=a \\
1 & \text { if } t \in(a, b],
\end{array} \quad g(t):=\left\{\begin{array}{cl}
-1 & \text { if } t \in[a, b) \\
0 & \text { if } t=b
\end{array}\right.\right.
$$

The functions $f$ and $g$ are monotonic nondecreasing. We will show that the Riemann-Stieltjes integral $\int_{a}^{b} f(t) d g(t)$ exists. 
Take the sequence of divisions and intermediate points

$$
d_{n}: a=x_{0}^{(n)}<\xi_{0}^{(n)}<x_{1}^{(n)}<\cdots<x_{n-1}^{(n)}<\xi_{n-1}^{(n)}<x_{n}^{(n)}=b
$$

such that $\Delta\left(d_{n}\right):=\max _{i \in\{0, \ldots, n-1\}}\left\{x_{i+1}^{(n)}-x_{i}^{(n)}\right\} \rightarrow 0$ as $n \rightarrow \infty$.

By the definition of the Riemann-Stieltjes integral $\int_{a}^{b} f(t) d g(t)$ we have

$$
\begin{aligned}
\int_{a}^{b} f(t) d g(t)= & \lim _{n \rightarrow \infty} \sum_{i=0}^{n-1} f\left(\xi_{i}^{(n)}\right)\left[g\left(x_{i+1}^{(n)}\right)-g\left(x_{i}^{(n)}\right)\right] \\
= & \lim _{n \rightarrow \infty} \sum_{i=0}^{n-2} f\left(\xi_{i}^{(n)}\right)\left[g\left(x_{i+1}^{(n)}\right)-g\left(x_{i}^{(n)}\right)\right] \\
& +\lim _{n \rightarrow \infty} f\left(\xi_{n-1}^{(n)}\right)\left[g(b)-g\left(x_{n-1}^{(n)}\right)\right] \\
= & 0+1=1 .
\end{aligned}
$$

Now, define the function $\ell:[a, b] \rightarrow \mathbb{R}$ by

$$
\ell(t):=f(t) g(t)=\left\{\begin{array}{cl}
0 & \text { if } t=a \\
-1 & \text { if } t \in(a, b) \\
0 & \text { if } t=a .
\end{array}\right.
$$

For a continuous function $h:[a, b] \rightarrow \mathbb{R}$, since $\ell$ is of bounded variation, then the integral $\int_{a}^{b} h(t) d \ell(t)$ exists.

Take the sequence of divisions and intermediate points

$$
d_{n}: a=x_{0}^{(n)}<\xi_{0}^{(n)}<x_{1}^{(n)}<\cdots<x_{n-1}^{(n)}<\xi_{n-1}^{(n)}<x_{n}^{(n)}=b
$$

such that $\Delta\left(d_{n}\right):=\max _{i \in\{0, \ldots, n-1\}}\left\{x_{i+1}^{(n)}-x_{i}^{(n)}\right\} \rightarrow 0$ as $n \rightarrow \infty$. Then we have

$$
\begin{aligned}
\int_{a}^{b} h(t) d \ell(t)= & \lim _{n \rightarrow \infty} \sum_{i=0}^{n-1} h\left(\xi_{i}^{(n)}\right)\left[\ell\left(x_{i+1}^{(n)}\right)-\ell\left(x_{i}^{(n)}\right)\right] \\
= & \lim _{n \rightarrow \infty} h\left(\xi_{0}^{(n)}\right)\left[\ell\left(x_{1}^{(n)}\right)-\ell(a)\right] \\
& +\lim _{n \rightarrow \infty} \sum_{i=1}^{n-2} h\left(\xi_{i}^{(n)}\right)\left[\ell\left(x_{i+1}^{(n)}\right)-\ell\left(x_{i}^{(n)}\right)\right] \\
& +\lim _{n \rightarrow \infty} h\left(\xi_{n-1}^{(n)}\right)\left[\ell(b)-\ell\left(x_{n-1}^{(n)}\right)\right] \\
= & \lim _{n \rightarrow \infty} h\left(\xi_{0}^{(n)}\right)(-1-0)+0+\lim _{n \rightarrow \infty} h\left(\xi_{n-1}^{(n)}\right)[0-(-1)] \\
= & h(b)-h(a) .
\end{aligned}
$$

Consider the functions $u, v:[a, b] \rightarrow \mathbb{R}$ given by

$$
u(t):=|f(t) h(t)|=\left\{\begin{array}{cl}
0 & \text { if } t=a \\
|h(t)| & \text { if } t \in(a, b],
\end{array}\right.
$$


and

$$
v(t):=|g(t) h(t)|=\left\{\begin{array}{cl}
|h(t)| & \text { if } t \in[a, b) \\
0 & \text { if } t=b .
\end{array}\right.
$$

Take the sequence of divisions and intermediate points

$$
d_{n}: a=x_{0}^{(n)}<\xi_{0}^{(n)}<x_{1}^{(n)}<\cdots<x_{n-1}^{(n)}<\xi_{n-1}^{(n)}<x_{n}^{(n)}=b
$$

such that $\Delta\left(d_{n}\right):=\max _{i \in\{0, \ldots, n-1\}}\left\{x_{i+1}^{(n)}-x_{i}^{(n)}\right\} \rightarrow 0$ as $n \rightarrow \infty$. Then we have

$$
\begin{aligned}
\int_{a}^{b} u(t) d g(t) & =\lim _{n \rightarrow \infty} \sum_{i=0}^{n-1} u\left(\xi_{i}^{(n)}\right)\left[g\left(x_{i+1}^{(n)}\right)-g\left(x_{i}^{(n)}\right)\right] \\
& =\lim _{n \rightarrow \infty} u\left(\xi_{n-1}^{(n)}\right)\left[g(b)-g\left(x_{n-1}^{(n)}\right)\right] \\
& =\lim _{n \rightarrow \infty}\left|h\left(\xi_{n-1}^{(n)}\right)\right|[0-(-1)]=|h(b)|
\end{aligned}
$$

and

$$
\begin{aligned}
\int_{a}^{b} v(t) d f(t) & =\lim _{n \rightarrow \infty} \sum_{i=0}^{n-1} v\left(\xi_{i}^{(n)}\right)\left[f\left(x_{i+1}^{(n)}\right)-f\left(x_{i}^{(n)}\right)\right] \\
& =\lim _{n \rightarrow \infty} v\left(\xi_{0}^{(n)}\right)\left[f\left(x_{1}^{(n)}\right)-f(a)\right] \\
& =\lim _{n \rightarrow \infty}\left|h\left(\xi_{0}^{(n)}\right)\right|(1-0)=|h(a)| .
\end{aligned}
$$

Replacing these values in (2.15) we have

$$
|h(b)-h(a)| \leq|h(b)|+|h(a)| .
$$

This inequality reduces to an equality if we choose a continuous function $h$ : $[a, b] \rightarrow \mathbb{R}$ such that $h(b)=-h(a)$. For instance, for $h:[a, b] \rightarrow \mathbb{R}, h(t)=$ $t-\frac{a+b}{2}$, we get in both sides of (2.17) the same quantity $b-a$.

Remark 4. We remark that in the dual case, namely when the functions $f, g$ : $[a, b] \rightarrow \mathbb{C}$ are continuous and $h:[a, b] \rightarrow \mathbb{R}$ is monotonic nondecreasing, then the Riemann-Stieltjes integral $\int_{a}^{b} h(t) d(f(t) g(t))$ exists and integrating by parts we have the equality (2.14). Taking the modulus in this equality and using the property $(2.16)$ we get

$$
\begin{aligned}
& \left|f(b) g(b) h(b)-f(a) g(a) h(a)-\int_{a}^{b} h(t) d(f(t) g(t))\right| \\
\leq & \int_{a}^{b}|f(t) g(t)| d(h(t)) .
\end{aligned}
$$

We observe that in our Theorems 1, 2 and 3 we assumed that the factors of the integrator, namely $f$ and $g$ have the same properties. The reader can also consider the other cases when one has a property and the other has a different 
property, for example $f$ is of bounded variation and $g$ is Lipschitzian with a constant $L$. In this case, if $h$ is continuous, then we have

$$
\begin{aligned}
& \left|\int_{a}^{b} h(t) d(f(t) g(t))\right| \\
\leq & \left|\int_{a}^{b} h(t) f(t) d(g(t))\right|+\left|\int_{a}^{b} h(t) g(t) d(f(t))\right| \\
\leq & L\left|\int_{a}^{b} h(t) f(t) d t\right|+\sup _{t \in[a, b]}|h(t) g(t)| \bigvee_{a}^{b}(f) .
\end{aligned}
$$

The details in the other cases are omitted.

\section{Applications for trapezoid type inequalities}

The following result holds:

Proposition 1. Let $f, g:[a, b] \rightarrow \mathbb{C}$ be two functions of bounded variation and such that the Riemann-Stieltjes integral $\int_{a}^{b} f(t) d g(t)$ exists. Then for any $x \in[a, b]$ we have

$$
\begin{aligned}
& \left|f(b) g(b)(b-x)+f(a) g(a)(x-a)-\int_{a}^{b} f(t) g(t) d t\right| \\
\leq & \sup _{t \in[a, b]}|(t-x) g(t)| \bigvee_{a}^{b}(f)+\sup _{t \in[a, b]}|(t-x) f(t)| \bigvee_{a}^{b}(g) \\
\leq & {\left[\frac{1}{2}(b-a)+\left|x-\frac{a+b}{2}\right|\right]\left[\|g\|_{\infty} \bigvee_{a}^{b}(f)+\|f\|_{\infty} \bigvee_{a}^{b}(g)\right] . }
\end{aligned}
$$

In particular, we have

$$
\begin{aligned}
& \left|\frac{f(b) g(b)+f(a) g(a)}{2}(b-a)-\int_{a}^{b} f(t) g(t) d t\right| \\
\leq & \sup _{t \in[a, b]}\left|\left(t-\frac{a+b}{2}\right) g(t)\right| \bigvee_{a}^{b}(f)+\sup _{t \in[a, b]}\left|\left(t-\frac{a+b}{2}\right) f(t)\right| \bigvee_{a}^{b}(g) \\
\leq & \frac{1}{2}(b-a)\left[\|g\|_{\infty} \bigvee_{a}^{b}(f)+\|f\|_{\infty} \bigvee_{a}^{b}(g)\right] .
\end{aligned}
$$

The inequalities (3.2) are sharp.

Proof. We use the following identity

$$
F(b)(b-x)+F(a)(x-a)-\int_{a}^{b} F(t) d t=\int_{a}^{b}(t-x) d F(t)
$$


that holds for any function of bounded variation $F:[a, b] \rightarrow \mathbb{C}$ and any $x \in$ $[a, b]$.

If we write the equality (3.3) for $F=f g$ we get

$$
\begin{aligned}
& f(b) g(b)(b-x)+f(a) g(a)(x-a)-\int_{a}^{b} f(t) g(t) d t \\
= & \int_{a}^{b}(t-x) d(f(t) g(t))
\end{aligned}
$$

for any $x \in[a, b]$.

If we use Theorem 1 for the function $h(t)=t-x, t \in[a, b]$, then we have the inequality

$$
\begin{aligned}
& \left|\int_{a}^{b}(t-x) d(f(t) g(t))\right| \\
\leq & \sup _{t \in[a, b]}|(t-x) g(t)| \bigvee_{a}^{b}(f)+\sup _{t \in[a, b]}|(t-x) g(f)| \bigvee_{a}^{b}(g) \\
\leq & \sup _{t \in[a, b]}|t-x|\left[\|g\|_{\infty} \bigvee_{a}^{b}(f)+\|f\|_{\infty} \bigvee_{a}^{b}(g)\right] \\
= & \max _{a}\{x-a, b-x\}\left[\|g\|_{\infty} \bigvee_{a}^{b}(f)+\|f\|_{\infty} \bigvee_{a}^{b}(g)\right] \\
= & {\left[\frac{1}{2}(b-a)+\left|x-\frac{a+b}{2}\right|\right]\left[\|g\|_{\infty} \bigvee_{a}^{b}(f)+\|f\|_{\infty} \bigvee_{a}^{b}(g)\right] }
\end{aligned}
$$

and the desired result (3.1) is obtained.

The inequality (3.2) follows from (3.1) for $x=\frac{a+b}{2}$.

Let us prove the sharpness of the inequalities (3.2).

Consider the functions $f, g:[a, b] \rightarrow \mathbb{R}$ defined by

$$
f(t):=\left\{\begin{array}{ll}
0 & \text { if } t=a \\
1 & \text { if } t \in(a, b],
\end{array} \quad g(t):= \begin{cases}1 & \text { if } t \in[a, b) \\
0 & \text { if } t=b .\end{cases}\right.
$$

We observe that $f$ and $g$ are of bounded variation and

$$
\bigvee_{a}^{b}(f)=\bigvee_{a}^{b}(g)=1
$$

Take the sequence of divisions and intermediate points

$$
d_{n}: a=x_{0}^{(n)}<\xi_{0}^{(n)}<x_{1}^{(n)}<\cdots<x_{n-1}^{(n)}<\xi_{n-1}^{(n)}<x_{n}^{(n)}=b
$$

such that $\Delta\left(d_{n}\right):=\max _{i \in\{0, \ldots, n-1\}}\left\{x_{i+1}^{(n)}-x_{i}^{(n)}\right\} \rightarrow 0$ as $n \rightarrow \infty$. 
By the definition of the Riemann-Stieltjes integral $\int_{a}^{b} f(t) d g(t)$ we have

$$
\begin{aligned}
\int_{a}^{b} f(t) d g(t)= & \lim _{n \rightarrow \infty} \sum_{i=0}^{n-1} f\left(\xi_{i}^{(n)}\right)\left[g\left(x_{i+1}^{(n)}\right)-g\left(x_{i}^{(n)}\right)\right] \\
= & \lim _{n \rightarrow \infty} \sum_{i=0}^{n-2} f\left(\xi_{i}^{(n)}\right)\left[g\left(x_{i+1}^{(n)}\right)-g\left(x_{i}^{(n)}\right)\right] \\
& +\lim _{n \rightarrow \infty} f\left(\xi_{n-1}^{(n)}\right)\left[g(b)-g\left(x_{n-1}^{(n)}\right)\right] \\
= & 0-1=-1
\end{aligned}
$$

which shows that this integral exists.

Observe that

$$
\begin{aligned}
& \left(t-\frac{a+b}{2}\right) f(t):=\left\{\begin{array}{cl}
0 & \text { if } t=a \\
t-\frac{a+b}{2} & \text { if } t \in(a, b],
\end{array}\right. \\
& \left(t-\frac{a+b}{2}\right) g(t):=\left\{\begin{array}{cl}
t-\frac{a+b}{2} & \text { if } t \in[a, b) \\
0 & \text { if } t=b .
\end{array}\right.
\end{aligned}
$$

Then

$$
\sup _{t \in[a, b]}\left|\left(t-\frac{a+b}{2}\right) g(t)\right|=\frac{b-a}{2}
$$

and

$$
\sup _{t \in[a, b]}\left|\left(t-\frac{a+b}{2}\right) f(t)\right|=\frac{b-a}{2} .
$$

We also have

$$
\frac{f(b) g(b)+f(a) g(a)}{2}(b-a)-\int_{a}^{b} f(t) g(t) d t=-(b-a) .
$$

Now, if we replace these values in (3.2) then we get in all terms the same quantity $b-a$.

In a similar way we can prove the following results as well:

Proposition 2. Let $f:[a, b] \rightarrow \mathbb{C}$ be Lipschitzian with the constant $L>0$ and $g:[a, b] \rightarrow \mathbb{C}$ be Lipschitzian with the constant $K>0$. Then for any $x \in[a, b]$ we have

$$
\begin{aligned}
& \left|f(b) g(b)(b-x)+f(a) g(a)(x-a)-\int_{a}^{b} f(t) g(t) d t\right| \\
\leq & L \int_{a}^{b}|t-x||g(t)| d t+K \int_{a}^{b}|t-x||f(t)| d t \\
\leq & {\left[\frac{1}{4}(b-a)^{2}+\left(x-\frac{a+b}{2}\right)^{2}\right]\left(L\|g\|_{\infty}+K\|f\|_{\infty}\right) . }
\end{aligned}
$$


In particular, we have

$$
\begin{aligned}
& \left|\frac{f(b) g(b)+f(a) g(a)}{2}(b-a)-\int_{a}^{b} f(t) g(t) d t\right| \\
\leq & L \int_{a}^{b}\left|t-\frac{a+b}{2}\right||g(t)| d t+K \int_{a}^{b}\left|t-\frac{a+b}{2}\right||f(t)| d t \\
\leq & \frac{1}{4}(b-a)^{2}\left(L\|g\|_{\infty}+K\|f\|_{\infty}\right) .
\end{aligned}
$$

We also have:

Proposition 3. Assume that $f, g:[a, b] \rightarrow \mathbb{R}$ are monotonic nondecreasing on $[a, b]$ and such that the Riemann-Stieltjes integral $\int_{a}^{b} f(t) d g(t)$ exists. Then for any $x \in[a, b]$ we have

$$
\begin{aligned}
& \left|f(b) g(b)(b-x)+f(a) g(a)(x-a)-\int_{a}^{b} f(t) g(t) d t\right| \\
\leq & \int_{a}^{b}|t-x||g(t)| d f(t)+\int_{a}^{b}|t-x||f(t)| d g(t) \\
\leq & {\left[\frac{1}{2}(b-a)+\left|x-\frac{a+b}{2}\right|\right]\left(\int_{a}^{b}|g(t)| d f(t)+\int_{a}^{b}|f(t)| d g(t)\right) . }
\end{aligned}
$$

In particular, we have

$$
\begin{aligned}
& \left|\frac{f(b) g(b)+f(a) g(a)}{2}(b-a)-\int_{a}^{b} f(t) g(t) d t\right| \\
\leq & \int_{a}^{b}\left|t-\frac{a+b}{2}\right||g(t)| d f(t)+\int_{a}^{b}\left|t-\frac{a+b}{2}\right||f(t)| d g(t) \\
\leq & \frac{1}{2}(b-a)\left(\int_{a}^{b}|g(t)| d f(t)+\int_{a}^{b}|f(t)| d g(t)\right) .
\end{aligned}
$$

\section{Applications for Ostrowski type inequalities}

The following result holds:

Proposition 4. Let $f, g:[a, b] \rightarrow \mathbb{C}$ be two functions of bounded variation and such that for $x \in(a, b)$ the Riemann-Stieltjes integrals $\int_{a}^{x} f(t) d g(t)$ and $\int_{x}^{b} f(t) d g(t)$ exist. Then we have

$$
\begin{aligned}
& \left|f(x) g(x)(b-a)-\int_{a}^{b} f(t) g(t) d t\right| \\
\leq & (x-a) \sup _{t \in[a, x]}\{|f(t)|\} \bigvee_{a}^{x}(g)+(x-a) \sup _{t \in[a, x]}\{|g(t)|\} \bigvee_{a}^{x}(f)
\end{aligned}
$$




$$
\begin{aligned}
& +(b-x) \sup _{t \in[x, b]}\{|f(t)|\} \bigvee_{x}^{b}(g)+(b-x) \sup _{t \in[x, b]}\{|g(t)|\} \bigvee_{x}^{b}(f) \\
\leq & {\left[\frac{1}{2}(b-a)+\left|x-\frac{a+b}{2}\right|\right]\left[\|g\|_{\infty} \bigvee_{a}^{b}(f)+\|f\|_{\infty} \bigvee_{a}^{b}(g)\right] . }
\end{aligned}
$$

In particular, if the Riemann-Stieltjes integrals $\int_{a}^{\frac{a+b}{2}} f(t) d g(t)$ and $\int_{\frac{a+b}{2}}^{b} f(t) d g(t)$ exist, then we have

$$
\begin{aligned}
& \left|f\left(\frac{a+b}{2}\right) g\left(\frac{a+b}{2}\right)(b-a)-\int_{a}^{b} f(t) g(t) d t\right| \\
\leq & \frac{b-a}{2}\left[\sup _{t \in\left[a, \frac{a+b}{2}\right]}\{|f(t)|\} \bigvee_{a}^{\frac{a+b}{2}}(g)+\sup _{t \in\left[a, \frac{a+b}{2}\right]}\{|g(t)|\} \bigvee_{a}^{\frac{a+b}{2}}(f)\right. \\
& \left.\sup _{t \in\left[\frac{a+b}{2}, b\right]}\{|f(t)|\} \bigvee_{\frac{a+b}{2}}^{b}(g)+\sup _{t \in\left[\frac{a+b}{2}, b\right]}\{|g(t)|\} \bigvee_{\frac{a+b}{2}}^{b}(f)\right] \\
\leq & \frac{1}{2}(b-a)\left[\|g\|_{\infty} \bigvee_{a}^{b}(f)+\|f\|_{\infty} \bigvee_{a}^{b}(g)\right] .
\end{aligned}
$$

The inequalities in (4.2) are sharp.

Proof. We use the following identity (see for instance [23])

$$
F(x)(b-a)-\int_{a}^{b} F(t) d t=\int_{a}^{x}(t-a) d F(t)+\int_{x}^{b}(t-b) d F(t)
$$

that holds for any function of bounded variation $F:[a, b] \rightarrow \mathbb{C}$ and any $x \in$ $[a, b]$.

If we write the equality (4.3) for $F=f g$ we get

$$
\begin{aligned}
& f(x) g(x)(b-a)-\int_{a}^{b} f(t) g(t) d t \\
= & \int_{a}^{x}(t-a) d(f(t) g(t))+\int_{x}^{b}(t-b) d(f(t) g(t))
\end{aligned}
$$

for any functions $f, g:[a, b] \rightarrow \mathbb{C}$ of bounded variation and any $x \in[a, b]$.

Taking the modulus on (4.4) and utilizing Theorem 1 on the intervals $[a, x]$ and $[x, b]$ we have successively that

$$
\begin{aligned}
& \left|f(x) g(x)(b-a)-\int_{a}^{b} f(t) g(t) d t\right| \\
\leq & \left|\int_{a}^{x}(t-a) d(f(t) g(t))\right|+\left|\int_{x}^{b}(t-b) d(f(t) g(t))\right|
\end{aligned}
$$


INEQUALITIES FOR THE RIEMANN-STIELTJES INTEGRAL

$$
\begin{aligned}
\leq & \sup _{t \in[a, x]}\{(t-a)|f(t)|\} \bigvee_{a}^{x}(g)+\sup _{t \in[a, x]}\{(t-a)|g(t)|\} \bigvee_{a}^{x}(f) \\
& +\sup _{t \in[x, b]}\{(b-t)|f(t)|\} \bigvee_{x}^{b}(g)+\sup _{t \in[x, b]}\{(b-t)|g(t)|\} \bigvee_{x}^{b}(f) \\
\leq & (x-a) \sup _{t \in[a, x]}\{|f(t)|\} \bigvee_{a}^{x}(g)+(x-a) \sup _{t \in[a, x]}\{|g(t)|\} \bigvee_{a}^{x}(f) \\
& +(b-x) \sup _{t \in[x, b]}\{|f(t)|\} \bigvee_{x}^{b}(g)+(b-x) \sup _{t \in[x, b]}\{|g(t)|\} \bigvee_{x}^{b}(f) \\
\leq & \max \{x-a, b-x\} \sup _{t \in[a, b]}\{|f(t)|\} \bigvee_{a}^{b}(g) \\
& +\max \{x-a, b-x\} \sup _{t \in[a, b]}\{|g(t)|\} \bigvee_{a}^{b}(f) \\
= & {\left[\frac{1}{2}(b-a)+\left|x-\frac{a+b}{2}\right|\right]\left[\|g\|_{\infty} \bigvee_{a}^{b}(f)+\|f\|_{\infty} \bigvee_{a}^{b}(g)\right] }
\end{aligned}
$$

which proves the desired result (4.1).

The inequality (4.2) is obvious from (4.1).

Consider now the functions $f, g:[a, b] \rightarrow \mathbb{R}$ defined by

$$
f(t):=\left\{\begin{array}{ll}
0 & \text { if } t \in\left[a, \frac{a+b}{2}\right) \\
1 & \text { if } t \in\left[\frac{a+b}{2}, b\right]
\end{array} \quad g(t):= \begin{cases}1 & \text { if } t \in\left[a, \frac{a+b}{2}\right] \\
0 & \text { if } t \in\left(\frac{a+b}{2}, b\right] .\end{cases}\right.
$$

We observe that $f$ and $g$ are of bounded variation and

$$
\bigvee_{a}^{b}(f)=\bigvee_{a}^{b}(g)=1
$$

The Riemann-Stieltjes integrals $\int_{a}^{\frac{a+b}{2}} f(t) d g(t)$ and $\int_{\frac{a+b}{2}}^{b} f(t) d g(t)$ exist since one function is continuous while the other is of bounded variation on those intervals.

We observe that for these functions we have

$$
\begin{gathered}
f\left(\frac{a+b}{2}\right) g\left(\frac{a+b}{2}\right)(b-a)-\int_{a}^{b} f(t) g(t) d t=b-a, \\
\sup _{t \in\left[a, \frac{a+b}{2}\right]}\{|f(t)|\} \bigvee_{a}^{\frac{a+b}{2}}(g)+\sup _{t \in\left[a, \frac{a+b}{2}\right]}\{|g(t)|\} \bigvee_{a}^{\frac{a+b}{2}}(f)
\end{gathered}
$$




$$
+\sup _{t \in\left[\frac{a+b}{2}, b\right]}\{|f(t)|\} \bigvee_{\frac{a+b}{2}}^{b}(g)+\sup _{t \in\left[\frac{a+b}{2}, b\right]}\{|g(t)|\} \bigvee_{\frac{a+b}{2}}^{b}(f)=2
$$

and

$$
\|g\|_{\infty} \bigvee_{a}^{b}(f)+\|f\|_{\infty} \bigvee_{a}^{b}(g)=2
$$

Replacing these values in (4.2) we obtain in all terms the same quantity $b-a$, which proves the sharpness of the inequalities.

In a similar way we can prove the following results as well:

Proposition 5. Let $f:[a, b] \rightarrow \mathbb{C}$ be Lipschitzian with the constant $L>0$ and $g:[a, b] \rightarrow \mathbb{C}$ be Lipschitzian with the constant $K>0$. Then for any $x \in[a, b]$ we have

$$
\begin{aligned}
& \left|f(x) g(x)(b-a)-\int_{a}^{b} f(t) g(t) d t\right| \\
\leq & L\left(\int_{a}^{x}(t-a)|g(t)| d t+\int_{x}^{b}(b-t)|g(t)| d t\right) \\
& +K\left(\int_{a}^{x}(t-a)|f(t)| d t+\int_{x}^{b}(b-t)|f(t)| d t\right) \\
\leq & {\left[\frac{1}{4}(b-a)^{2}+\left(x-\frac{a+b}{2}\right)^{2}\right]\left(L\|g\|_{\infty}+K\|f\|_{\infty}\right) . }
\end{aligned}
$$

In particular, we have

$$
\begin{aligned}
& \left|f\left(\frac{a+b}{2}\right) g\left(\frac{a+b}{2}\right)(b-a)-\int_{a}^{b} f(t) g(t) d t\right| \\
\leq & L\left(\int_{a}^{\frac{a+b}{2}}(t-a)|g(t)| d t+\int_{\frac{a+b}{2}}^{b}(b-t)|g(t)| d t\right) \\
& +K\left(\int_{a}^{\frac{a+b}{2}}(t-a)|f(t)| d t+\int_{\frac{a+b}{2}}^{b}(b-t)|f(t)| d t\right) \\
\leq & \frac{1}{4}(b-a)^{2}\left(L\|g\|_{\infty}+K\|f\|_{\infty}\right) .
\end{aligned}
$$

We also have:

Proposition 6. Assume that $f, g:[a, b] \rightarrow \mathbb{R}$ are monotonic nondecreasing on $[a, b]$ and such that for $x \in(a, b)$ the Riemann-Stieltjes integrals $\int_{a}^{x} f(t) d g(t)$ 
and $\int_{x}^{b} f(t) d g(t)$ exist. Then we have

$$
\begin{aligned}
& \left|f(x) g(x)(b-a)-\int_{a}^{b} f(t) g(t) d t\right| \\
\leq & \int_{a}^{x}(t-a)|g(t)| d f(t)+\int_{x}^{b}(b-t)|g(t)| d f(t) \\
& +\int_{a}^{x}(t-a)|f(t)| d g(t)+\int_{x}^{b}(b-t)|f(t)| d g(t) \\
\leq & {\left[\frac{1}{2}(b-a)+\left|x-\frac{a+b}{2}\right|\right]\left(\int_{a}^{b}|g(t)| d f(t)+\int_{a}^{b}|f(t)| d g(t)\right) . }
\end{aligned}
$$

In particular, if the Riemann-Stieltjes integrals $\int_{a}^{\frac{a+b}{2}} f(t) d g(t)$ and $\int_{\frac{a+b}{2}}^{b} f(t) d g(t)$ exist, then we have

$$
\begin{aligned}
& \left|f\left(\frac{a+b}{2}\right) g\left(\frac{a+b}{2}\right)(b-a)-\int_{a}^{b} f(t) g(t) d t\right| \\
\leq & \int_{a}^{\frac{a+b}{2}}(t-a)|g(t)| d f(t)+\int_{\frac{a+b}{2}}^{b}(b-t)|g(t)| d f(t) \\
& +\int_{a}^{\frac{a+b}{2}}(t-a)|f(t)| d g(t)+\int_{\frac{a+b}{2}}^{b}(b-t)|f(t)| d g(t) \\
\leq & \frac{1}{2}(b-a)\left(\int_{a}^{b}|g(t)| d f(t)+\int_{a}^{b}|f(t)| d g(t)\right) .
\end{aligned}
$$

\section{Applications for selfadjoint operators}

We denote by $\mathcal{B}(H)$ the Banach algebra of all bounded linear operators on a complex Hilbert space $(H ;\langle\cdot, \cdot\rangle)$. Let $A \in \mathcal{B}(H)$ be selfadjoint and let $\varphi_{\lambda}$ be defined for all $\lambda \in \mathbb{R}$ as follows

$$
\varphi_{\lambda}(s):=\left\{\begin{array}{l}
1, \text { for }-\infty<s \leq \lambda, \\
0, \text { for } \lambda<s<+\infty .
\end{array}\right.
$$

Then for every $\lambda \in \mathbb{R}$ the operator

$$
E_{\lambda}:=\varphi_{\lambda}(A)
$$

is a projection which reduces $A$.

The properties of these projections are collected in the following fundamental result concerning the spectral representation of bounded selfadjoint operators in Hilbert spaces, see for instance [33, p. 256]:

Theorem 4 (Spectral Representation Theorem). Let $A$ be a bounded selfadjoint operator on the Hilbert space $H$ and let $m=\min \{\lambda \mid \lambda \in S p(A)\}=$ : 
$\min S p(A)$ and $M=\max \{\lambda \mid \lambda \in S p(A)\}=: \max S p(A)$. Then there exists a family of projections $\left\{E_{\lambda}\right\}_{\lambda \in \mathbb{R}}$, called the spectral family of $A$, with the following properties

a) $E_{\lambda} \leq E_{\lambda^{\prime}}$ for $\lambda \leq \lambda^{\prime}$

b) $E_{m-0}=0, E_{M}=I$ and $E_{\lambda+0}=E_{\lambda}$ for all $\lambda \in \mathbb{R}$;

c) We have the representation

$$
A=\int_{m-0}^{M} \lambda d E_{\lambda}
$$

More generally, for every continuous complex-valued function $\varphi$ defined on $\mathbb{R}$ and for every $\varepsilon>0$ there exists $a \delta>0$ such that

$$
\left\|\varphi(A)-\sum_{k=1}^{n} \varphi\left(\lambda_{k}^{\prime}\right)\left[E_{\lambda_{k}}-E_{\lambda_{k-1}}\right]\right\| \leq \varepsilon
$$

whenever

$$
\left\{\begin{array}{l}
\lambda_{0}<m=\lambda_{1}<\cdots<\lambda_{n-1}<\lambda_{n}=M \\
\lambda_{k}-\lambda_{k-1} \leq \delta \text { for } 1 \leq k \leq n \\
\lambda_{k}^{\prime} \in\left[\lambda_{k-1}, \lambda_{k}\right] \text { for } 1 \leq k \leq n
\end{array}\right.
$$

this means that

$$
\varphi(A)=\int_{m-0}^{M} \varphi(\lambda) d E_{\lambda},
$$

where the integral is of Riemann-Stieltjes type.

Corollary 2. With the assumptions of Theorem 4 for $A, E_{\lambda}$ and $\varphi$ we have the representations

$$
\varphi(A) x=\int_{m-0}^{M} \varphi(\lambda) d E_{\lambda} x \quad \text { for all } x \in H
$$

and

$$
\langle\varphi(A) x, y\rangle=\int_{m-0}^{M} \varphi(\lambda) d\left\langle E_{\lambda} x, y\right\rangle \quad \text { for all } x, y \in H .
$$

In particular,

$$
\langle\varphi(A) x, x\rangle=\int_{m-0}^{M} \varphi(\lambda) d\left\langle E_{\lambda} x, x\right\rangle \quad \text { for all } x \in H .
$$

Moreover, we have the equality

$$
\|\varphi(A) x\|^{2}=\int_{m-0}^{M}|\varphi(\lambda)|^{2} d\left\|E_{\lambda} x\right\|^{2} \quad \text { for all } x \in H .
$$

Proposition 7. Let $A$ be a bounded selfadjoint operator on the Hilbert space $H$ and let $m=\min \{\lambda \mid \lambda \in S p(A)\}=: \min S p(A)$ and $M=\max \{\lambda \mid \lambda \in S p(A)\}$ $=: \max S p(A)$. Assume that $f, g: \mathbb{R} \rightarrow \mathbb{C}$ are continuous. 
(i) If $f, g$ are of bounded variation on $[m, M]$, then

$$
\begin{aligned}
& |\langle[f(M) g(M) I-f(A) g(A)] x, y\rangle| \\
\leq & \sup _{t \in[m, M]}\left|\left\langle E_{t} x, y\right\rangle\right|\left[\sup _{t \in[m, M]}|f(t)| \bigvee_{m}^{M}(g)+\sup _{t \in[m, M]}|f(g)| \bigvee_{m}^{M}(f)\right] \\
\leq & \|x\|\|y\|\left[\sup _{t \in[m, M]}|f(t)| \bigvee_{m}^{M}(g)+\sup _{t \in[m, M]}|f(g)| \bigvee_{m}^{M}(f)\right]
\end{aligned}
$$

for any $x, y \in H$.

(ii) If $f, g: \mathbb{R} \rightarrow \mathbb{C}$ are Lipschitzian with the constants $L$, respectively $K$ on $[m, M]$, then

$$
\begin{aligned}
& |\langle[f(M) g(M) I-f(A) g(A)] x, y\rangle| \\
\leq & K \int_{m-0}^{M}\left|\left\langle E_{t} x, y\right\rangle f(t)\right| d t+L \int_{m-0}^{M}\left|\left\langle E_{t} x, y\right\rangle g(t)\right| d t \\
\leq & \sup _{t \in[m, M]}\left|\left\langle E_{t} x, y\right\rangle\right|\left[K \int_{m}^{M}|f(t)| d t+L \int_{m}^{M}|g(t)| d t\right] \\
\leq & \|x\|\|y\|\left[K \int_{m}^{M}|f(t)| d t+L \int_{m}^{M}|g(t)| d t\right]
\end{aligned}
$$

for any $x, y \in H$.

(iii) If $f, g: \mathbb{R} \rightarrow \mathbb{C}$ are monotonic nondecreasing on $[m, M]$, then

$$
\begin{aligned}
& |\langle[f(M) g(M) I-f(A) g(A)] x, y\rangle| \\
\leq & \int_{m-0}^{M}\left|\left\langle E_{t} x, y\right\rangle f(t)\right| d g(t)+\int_{m-0}^{M}\left|\left\langle E_{t} x, y\right\rangle g(t)\right| d f(t) \\
\leq & \sup _{t \in[m, M]}\left|\left\langle E_{t} x, y\right\rangle\right|\left[\int_{m}^{M}|f(t)| d g(t)+\int_{m}^{M}|g(t)| d f(t)\right] \\
\leq & \|x\|\|y\|\left[\int_{m}^{M}|f(t)| d g(t)+\int_{m}^{M}|g(t)| d f(t)\right]
\end{aligned}
$$

for any $x, y \in H$.

Proof. Let $\varepsilon>0$. We use the fact that (see Remark 1)

$$
\begin{aligned}
& \int_{m-\varepsilon}^{M}\left\langle E_{t} x, y\right\rangle d(f(t) g(t)) \\
= & \int_{m-\varepsilon}^{M}\left\langle E_{t} x, y\right\rangle f(t) d(g(t))+\int_{m-\varepsilon}^{M}\left\langle E_{t} x, y\right\rangle g(t) d(f(t))
\end{aligned}
$$

for any $x, y \in H$, since $\langle E . x, y\rangle$ is of bounded variation on $[m-\varepsilon, M]$ while $f$, $g$ are continuous on $[m-\varepsilon, M]$. 
Integrating by parts in the Riemann-Stieltjes integral, we also have

$$
\begin{aligned}
& \int_{m-\varepsilon}^{M}\left\langle E_{t} x, y\right\rangle d(f(t) g(t)) \\
= & \left.\left\langle E_{t} x, y\right\rangle(f(t) g(t))\right|_{m-\varepsilon} ^{M}-\int_{m-\varepsilon}^{M} f(t) g(t) d\left(\left\langle E_{t} x, y\right\rangle\right) \\
= & f(M) g(M)\langle x, y\rangle-\int_{m-\varepsilon}^{M} f(t) g(t) d\left(\left\langle E_{t} x, y\right\rangle\right)
\end{aligned}
$$

for any $x, y \in H$.

From (5.7) and (5.8) we then have

$$
\begin{aligned}
& f(M) g(M)\langle x, y\rangle-\int_{m-\varepsilon}^{M} f(t) g(t) d\left(\left\langle E_{t} x, y\right\rangle\right) \\
= & \int_{m-\varepsilon}^{M}\left\langle E_{t} x, y\right\rangle f(t) d(g(t))+\int_{m-\varepsilon}^{M}\left\langle E_{t} x, y\right\rangle g(t) d(f(t))
\end{aligned}
$$

for any $x, y \in H$ and $\varepsilon>0$.

(i). If $f$ and $g$ are of bounded variation, then by (5.9) we have

$$
\begin{aligned}
& \left|f(M) g(M)\langle x, y\rangle-\int_{m-\varepsilon}^{M} f(t) g(t) d\left(\left\langle E_{t} x, y\right\rangle\right)\right| \\
\leq & \left|\int_{m-\varepsilon}^{M}\left\langle E_{t} x, y\right\rangle f(t) d(g(t))\right|+\left|\int_{m-\varepsilon}^{M}\left\langle E_{t} x, y\right\rangle g(t) d(f(t))\right| \\
\leq & \sup _{t \in[m-\varepsilon, M]}\left|\left\langle E_{t} x, y\right\rangle f(t)\right| \bigvee_{m-\varepsilon}^{M}(g)+\sup _{t \in[m-\varepsilon, M]}\left|\left\langle E_{t} x, y\right\rangle g(t)\right| \bigvee_{m-\varepsilon}^{M}(f) \\
\leq & \sup _{t \in[m-\varepsilon, M]}\left|\left\langle E_{t} x, y\right\rangle\right| \sup _{t \in[m-\varepsilon, M]}|f(t)| \bigvee_{m-\varepsilon}^{M}(g) \\
& +\sup _{t \in[m-\varepsilon, M]}\left|\left\langle E_{t} x, y\right\rangle\right| \sup _{t \in[m-\varepsilon, M]}^{M}|g(t)| \bigvee_{m-\varepsilon}(f)
\end{aligned}
$$

for any $x, y \in H$ and $\varepsilon>0$.

Taking the limit over $\varepsilon \rightarrow 0+$ in (5.10) and utilizing the representation (5.3) we deduce (5.4).

The statements (ii) and (iii) can be proved in a similar way, however the details are omitted.

Acknowledgement. The author would like to thank the anonymous referee for valuable suggestions that have been implemented in the final version of the paper. 


\section{References}

[1] A. M. Acu, A. Baboş, and F. Sofonea, The mean value theorems and inequalities of Ostrowski type, Sci. Stud. Res. Ser. Math. Inform. 21 (2011), no. 1, 5-16.

[2] A. M. Acu and F. Sofonea, On an inequality of Ostrowski type, J. Sci. Arts 2011 (2011), no. 3(16), 281-287.

[3] F. Ahmad, N. S. Barnett, and S. S. Dragomir, New weighted Ostrowski and Čebyšev type inequalities, Nonlinear Anal. 71 (2009), no. 12, e1408-e1412.

[4] M. W. Alomari, A companion of Ostrowski's inequality with applications, Transylv. J. Math. Mech. 3 (2011), no. 1, 9-14.

[5] M. W. Alomari, M. Darus, S. S. Dragomir, and P. Cerone, Ostrowski type inequalities for functions whose derivatives are s-convex in the second sense, Appl. Math. Lett. 23 (2010), no. 9, 1071-1076.

[6] G. A. Anastassiou, Ostrowski type inequalities, Proc. Amer. Math. Soc. 123 (1995), no. $12,3775-3781$

[7] _ Univariate Ostrowski inequalities, revisited. Monatsh. Math. 135 (2002), no. 3, 175-189.

[8] _ Ostrowski inequalities for cosine and sine operator functions, Mat. Vesnik 64 (2012), no. 4, 336-346.

[9] $\longrightarrow$ Multivariate right fractional Ostrowski inequalities, J. Appl. Math. Inform. 30 (2012), no. 3-4, 445-454.

[10] Univariate right fractional Ostrowski inequalities, Cubo 14 (2012), no. 1, 1-7.

[11] N. S. Barnett, W.-S. Cheung, S. S. Dragomir, and A. Sofo, Ostrowski and trapezoid type inequalities for the Stieltjes integral with Lipschitzian integrands or integrators, Comput. Math. Appl. 57 (2009), no. 2, 195-201.

[12] N. S. Barnett and S. S. Dragomir, A perturbed trapezoid inequality in terms of the fourth derivative, Korean J. Comput. Appl. Math. 9 (2002), no. 1, 45-60.

[13] _ Perturbed version of a general trapezoid inequality, Inequality theory and applications, Vol. 3, 1-12, Nova Sci. Publ., Hauppauge, NY, 2003.

[14] A perturbed trapezoid inequality in terms of the third derivative and applications, Inequality theory and applications, Vol. 5, 1-11, Nova Sci. Publ., New York, 2007.

[15] N. S. Barnett, S. S. Dragomir, and I. Gomm, A companion for the Ostrowski and the generalised trapezoid inequalities, Math. Comput. Modelling 50 (2009), no. 1-2, 179-187.

[16] P. Cerone, W.-S. Cheung, and S. S. Dragomir, On Ostrowski type inequalities for Stieltjes integrals with absolutely continuous integrands and integrators of bounded variation, Comput. Math. Appl. 54 (2007), no. 2, 183-191.

[17] P. Cerone and S. S. Dragomir, Midpoint-type rules from an inequalities point of view, Handbook of analytic-computational methods in applied mathematics, 135-200, Chapman \& Hall/CRC, Boca Raton, FL, 2000.

[18] - Trapezoidal-type rules from an inequalities point of view, Handbook of analyticcomputational methods in applied mathematics, 65-134, Chapman \& Hall/CRC, Boca Raton, FL, 2000.

[19] P. Cerone, S. S. Dragomir, and C. E. M. Pearce, A generalized trapezoid inequality for functions of bounded variation, Turkish J. Math. 24 (2000), no. 2, 147-163.

[20] X.-L. Cheung and J. Sun, A note on the perturbed trapezoid inequality, J. Inequal. Pure Appl. Math. 3 (2002), no. 2, Article 29, 7 pp. (electronic).

[21] S. S. Dragomir, The Ostrowski integral inequality for mappings of bounded variation, Bull. Austral. Math. Soc. 60 (1999), no. 3, 495-508.

[22] _ On the midpoint quadrature formula for mappings with bounded variation and applications, Kragujevac J. Math. 22 (2000), 13-19.

[23] _ On the Ostrowski's integral inequality for mappings with bounded variation and applications, Math. Inequal. Appl. 4 (2001), no. 1, 59-66. 
[24] - On the trapezoid quadrature formula and applications, Kragujevac J. Math. 23 (2001), 25-36.

[25] Some inequalities of midpoint and trapezoid type for the Riemann-Stieltjes integral, Proceedings of the Third World Congress of Nonlinear Analysts, Part 4 (Catania, 2000); Nonlinear Anal. 47 (2001), no. 4, 2333-2340.

[26] _ Improvements of Ostrowski and generalised trapezoid inequality in terms of the upper and lower bounds of the first derivative, Tamkang J. Math. 34 (2003), no. 3, 213-222.

[27] _ Refinements of the generalised trapezoid and Ostrowski inequalities for functions of bounded variation, Arch. Math. (Basel) 91 (2008), no. 5, 450-460.

[28] _ Some inequalities for continuous functions of selfadjoint operators in Hilbert spaces, Acta Math. Vietnamica, to appear; Preprint, RGMIA Res. Rep. Coll. 15 (2012), Art. 16. http://rgmia.org/v15.php.

[29] S. S. Dragomir, Y. J. Cho, and Y.-H. Kim, On the trapezoid inequality for the RiemannStieltjes integral with Hölder continuous integrands and bounded variation integrators, Inequality theory and applications, Vol. 5, 71-79, Nova Sci. Publ., New York, 2007.

[30] S. S. Dragomir and A. Mcandrew, On trapezoid inequality via a Grüss type result and applications, Tamkang J. Math. 31 (2000), no. 3, 193-201.

[31] S. S. Dragomir, J. Peçarić, and S. Wang, The unified treatment of trapezoid, Simpson, and Ostrowski type inequality for monotonic mappings and applications, Math. Comput. Modelling 31 (2000), no. 6-7, 61-70.

[32] H. Gunawan, A note on Dragomir-McAndrew's trapezoid inequalities, Tamkang J. Math. 33 (2002), no. 3, 241-244.

[33] G. Helmberg, Introduction to Spectral Theory in Hilbert Space, John Wiley \& Sons, Inc. -New York, 1969.

[34] A. I. Kechriniotis and N. D. Assimakis, Generalizations of the trapezoid inequalities based on a new mean value theorem for the remainder in Taylor's formula, J. Inequal. Pure Appl. Math. 7 (2006), no. 3, Article 90, 13 pp. (electronic).

[35] Z. Liu, Some inequalities of perturbed trapezoid type, J. Inequal. Pure Appl. Math. 7 (2006), no. 2, Article 47, 9 pp.

[36] _ Some Ostrowski type inequalities and applications, Vietnam J. Math. 37 (2009), no. $1,15-22$.

[37] Some companions of an Ostrowski type inequality and applications, J. Inequal. Pure Appl. Math. 10 (2009), no. 2, Article 52, 12 pp.

[38] _ New sharp bound for a general Ostrowski type inequality, Tamsui Oxf. J. Math. Sci. 26 (2010), no. 1, 53-59.

[39] _ A sharp general Ostrowski type inequality, Bull. Aust. Math. Soc. 83 (2011), no. 2, 189-209.

[40] _ A note on Ostrowski type inequalities related to some s-convex functions in the second sense, Bull. Korean Math. Soc. 49 (2012), no. 4, 775-785.

[41] W.-J. Liu, Q.-L. Xue, and J.-W. Dong, New generalization of perturbed trapezoid, midpoint inequalities and applications, Int. J. Pure Appl. Math. 41 (2007), no. 6, 761-768.

[42] M. Masjed-Jamei and S. S. Dragomir, A new generalization of the Ostrowski inequality and applications, Filomat 25 (2011), no. 1, 115-123.

[43] P. R. Mercer, Hadamard's inequality and trapezoid rules for the Riemann-Stieltjes integral, J. Math. Anal. Appl. 344 (2008), no. 2, 921-926.

[44] A. Mcd. Mercer, On perturbed trapezoid inequalities, J. Inequal. Pure Appl. Math. 7 (2006), no. 4, Article 118, 7 pp. (electronic).

[45] B. G. Pachpatte, A note on a trapezoid type integral inequality, Bull. Greek Math. Soc. 49 (2004), 85-90.

[46] New inequalities of Ostrowski and trapezoid type for $n$-time differentiable functions, Bull. Korean Math. Soc. 41 (2004), no. 4, 633-639. 
[47] J. Park, On the Ostrowskilike type integral inequalities for mappings whose second derivatives are $s^{*}$-convex, Far East J. Math. Sci. (FJMS) 67 (2012), no. 1, 21-35.

[48] _ Some Ostrowskilike type inequalities for differentiable real $(\alpha, m)$-convex mappings, Far East J. Math. Sci. (FJMS) 61 (2012), no. 1, 75-91

[49] M. Z. Sarikaya, On the Ostrowski type integral inequality, Acta Math. Univ. Comenian. (N.S.) 79 (2010), no. 1, 129-134.

[50] W. T. Sulaiman, Some new Ostrowski type inequalities, J. Appl. Funct. Anal. 7 (2012), no. 1-2, 102-107.

[51] K.-L. Tseng, Improvements of the Ostrowski integral inequality for mappings of bounded variation II, Appl. Math. Comput. 218 (2012), no. 10, 5841-5847.

[52] K.-L. Tseng, S.-R. Hwang, G.-S. Yang, and Y.-M. Chou, Improvements of the Ostrowski integral inequality for mappings of bounded variation I, Appl. Math. Comput. 217 (2010), no. 6, 2348-2355.

[53] N. Ujević, Perturbed trapezoid and mid-point inequalities and applications, Soochow J. Math. 29 (2003), no. 3, 249-257.

[54] - On perturbed mid-point and trapezoid inequalities and applications, Kyungpook Math. J. 43 (2003), no. 3, 327-334.

[55] — Error inequalities for a generalized trapezoid rule, Appl. Math. Lett. 19 (2006), no. $1,32-37$.

[56] S. W. Vong, A note on some Ostrowski-like type inequalities, Comput. Math. Appl. 62 (2011), no. 1, 532-535.

[57] Q. Wu and S. Yang, A note to Ujević's generalization of Ostrowski's inequality, Appl. Math. Lett. 18 (2005), no. 6, 657-665.

[58] Y. Wu and Y. Wang, On the optimal constants of Ostrowskilike inequalities involving $n$ knots, Appl. Math. Comput. 219 (2013), no. 14, 7789-7794.

[59] Y.-X. Xiao, Remarks on Ostrowskilike inequalities, Appl. Math. Comput. 219 (2012), no. $3,1158-1162$.

Mathematics, College of Engineering \& Science

Victoria University, PO Box 14428

Melbourne City, MC 8001, Australia

AND

School of Computational \& Applied Mathematics

University of THE WitwatersRand

Private Bag 3, Johannesburg 2050, South Africa

E-mail address: sever.dragomir@vu.edu.au 